This item was submitted to Loughborough's Research Repository by the author.

Items in Figshare are protected by copyright, with all rights reserved, unless otherwise indicated.

\title{
Maneuver of fixed-wing combat aircraft
}

PLEASE CITE THE PUBLISHED VERSION

http://dx.doi.org/10.1002/9780470686652.eae249

PUBLISHER

(c) John Wiley and Sons, Inc.

VERSION

NA (Not Applicable or Unknown)

LICENCE

CC BY-NC-ND 4.0

REPOSITORY RECORD

Render, Peter M.. 2011. "Maneuver of Fixed-wing Combat Aircraft". figshare. https://hdl.handle.net/2134/8031. 
This item was submitted to Loughborough's Institutional Repository (https://dspace.lboro.ac.uk/) by the author and is made available under the following Creative Commons Licence conditions.

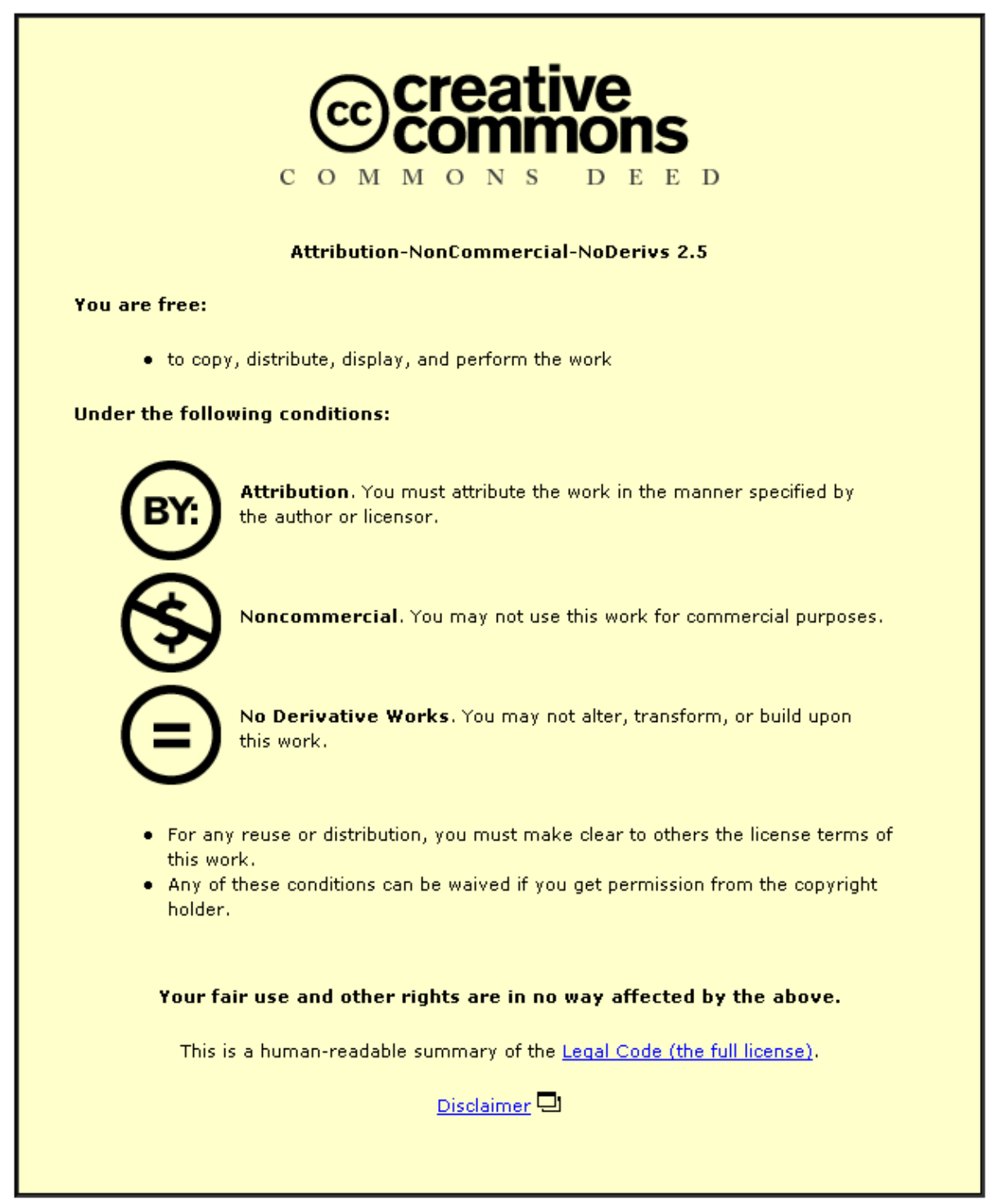

For the full text of this licence, please go to: http://creativecommons.org/licenses/by-nc-nd/2.5/ 


\section{Chapter eae249}

\section{Maneuver of Fixed-Wing Combat Aircraft}

\section{Peter M. Render}

Department of Aeronautical and Automotive Engineering, Loughborough University, Loughborough, England, UK

\author{
1 Introduction \\ 2 Energy Methods \\ 3 Climb Performance \\ 4 Turn Performance \\ 5 Performance Metrics \\ 6 New Technologies \\ 7 Conclusion \\ Acknowledgments \\ Notation
}

References

\section{INTRODUCTION}

The ability to maneuver is an important consideration for fixed wing combat aircraft and is required for both aggressive and evasive maneuvers. Small differences in maneuver performance can be significant in determining which aircraft will win a combat engagement. For example, pilots often regard a difference of $2-3^{\circ} \mathrm{s}^{-1}$ in turn rate as being significant. The required maneuver performance will be determined by the aircraft's role. For instance, an air combat aircraft designed to engage enemy fighters is likely to require high instantaneous turn rates, whilst a beyond visual range interceptor armed with long range missiles is more likely to be concerned with acceleration at high altitude and good climb to height characteristics.

Encyclopedia of Aerospace Engineering.

Edited by Richard Blockley and Wei Shyy

(C) 2010 John Wiley \& Sons, Ltd. ISBN: 978-0-470-75440-5

\section{ENERGY METHODS}

When initiating many maneuvers at fixed thrust, for example an instantaneous turn, an aircraft will slow down due to the increased drag. In other words, the aircraft is using energy. In combat situations energy management is important since, generally, the aircraft with the highest energy has the best chance of surviving.

\subsection{Specific energy, $h_{\mathrm{e}}$}

The total energy of an aircraft comprises kinetic and potential energy. Dividing the total energy by aircraft weight gives specific energy.

$$
h_{\mathrm{e}}=h_{\mathrm{z}}+\frac{1}{2 g} V^{2}
$$

where $h_{\mathrm{z}}$ is aircraft height, and $V$ is true air speed. Specific energy $h_{\mathrm{e}}$ is also known as energy height, since it has the units of length $(\mathrm{m})$.

\subsection{Specific excess power, $\boldsymbol{P}_{\mathrm{s}}$}

Noting that power is the rate of energy used per second, the specific power $P$ used by an aircraft during a maneuver can be defined as

$$
P=\frac{\mathrm{d} h_{\mathrm{e}}}{\mathrm{d} t}=\frac{\mathrm{d} h_{\mathrm{z}}}{\mathrm{d} t}+\frac{V}{g} \frac{\mathrm{d} V}{\mathrm{~d} t}
$$

From equation (2), for an aircraft to climb or accelerate without losing height, power is required. This power may 
come from the engine, but is reduced by the drag of the airframe. The difference between engine thrust and drag can be defined in terms of specific excess power, $P_{\mathrm{s}}$

$$
P_{\mathrm{s}}=\frac{(T-D) V}{W}
$$

Specific excess power represents the power available to an aircraft for use in maneuvers. This can be substituted for the specific power used, $P$, in equation (2), to determine the maneuver performance of an aircraft.

Equation (3) is identical to the equation developed for rate of climb $V_{\mathrm{c}}$ of a jet aircraft (see Volume 5, Chapter eae247). Thinking in terms of climb performance, for an aircraft flying at a constant forward speed $V$ and fixed thrust

when

$$
\begin{array}{lll}
P_{\mathrm{s}}>0 & V_{\mathrm{c}}>0 & \text { Aircraft climbs } \\
P_{\mathrm{s}}=0 & V_{\mathrm{c}}=0 & \text { Aircraft in level flight } \\
P_{\mathrm{s}}<0 & V_{\mathrm{c}}<0 & \text { Aircraft descends }
\end{array}
$$

In a trivial manner, the use of $P_{\mathrm{s}}$ to allow an aircraft's capability for maneuvering to be assessed has been illustrated. However, it should be noted that when $P_{\mathrm{s}}=0$ the aircraft can still climb, but its forward speed will reduce. Similarly the aircraft can accelerate, but only by descending at the same time.

The drag generated in a maneuver can be expressed as

$$
D=q S\left(C_{D_{0}}+K n^{2} C_{L_{\mathrm{i}}}^{2}\right)
$$

where $q$ is the dynamic pressure, $n$ the load factor and $C_{L_{\mathrm{i}}}$ is the lift coefficient immediately before initiating the maneuver. For a combat aircraft equation (4) represents a significant simplification of the drag characteristics since it assumes that the air flow remains attached, implying low angles of attack. In addition the effects of Mach number are also ignored.

Expressing $C_{L_{\mathrm{i}}}$ in terms of aircraft weight, $\mathrm{W}$, and substituting equation (4) into equation (3) gives

$$
P_{\mathrm{s}}=\left(\frac{T}{W}-\frac{q C_{D_{0}}}{W / S}-n^{2} \frac{K}{q} \frac{W}{S}\right)
$$

This is a useful equation since it illustrates the desirability of high thrust to weight ratio $(T / W)$ and the relative importance of profile drag (the $C_{D_{0}}$ term) and induced drag (the $n^{2}$ term). Induced drag becomes increasingly dominant as either speed is reduced or load factor is increased. Interestingly, in terms of specific excess power, high wing loading is beneficial at high speeds (high $q$ ), but detrimental at low speeds and high $n$, since it increases induced drag. Air-to-air combat is frequently initiated at high speed, but is then characterized by a loss of energy and a reduction in air speed as aircraft jostle for supremacy. Therefore, the low speed $P_{\mathrm{s}}$ characteristics of combat aircraft are vitally important.

Energy and specific excess power are useful concepts for comparing the maneuver potential of rival aircraft, and this is further considered in Section 5.1 .

\subsection{Maximum-energy-climb schedule}

Equation (2) showed that the specific power used by an aircraft is related to the rate of change of specific energy. Rearranging equation (2) gives

$$
\mathrm{d} t=\int_{h_{\mathrm{e}_{1}}}^{h_{\mathrm{e}_{2}}} \frac{\mathrm{d} h_{\mathrm{e}}}{P}
$$

which represents the time taken to change from one energy height $\left(h_{\mathrm{e}_{1}}\right)$ to another $\left(h_{\mathrm{e}_{2}}\right)$. In terms of the specific power available, equation (6) suggests that to minimize the time taken, the specific excess power $P_{\mathrm{s}}$ at each energy height the aircraft passes through should be maximized. This is called a maximum-energy-climb schedule. For a given aircraft, equation (5) can be used to generate contours of constant $P_{\mathrm{s}}$. Such contours are shown in Figure 1 plotted against altitude and Mach number. Also shown in Figure 1 are lines of constant energy height. This figure can now be used to determine the required flight profile (or energy climb schedule) that minimizes the time taken to maneuver between any two points. Assume that an aircraft is at Point A and wishes to carry out an attack at Point B. To minimize the time, $P_{\mathrm{s}}$ has to be maximized at each energy height. Mathematically this can be shown to occur at points where the $P_{\mathrm{s}}$ contours are tangential to the $h_{\mathrm{e}}$ curves. The maximum-energy-climb schedule passes through these points. To achieve this, the aircraft starting at $\mathrm{A}$ initiates a dive to follow the line of constant energy height to Point $\mathrm{C}$. The aircraft then starts to climb along a flight path which passes through the points where the lines of constant energy height are tangential to the $P_{\mathrm{s}}$ contours. At Point $\mathrm{D}$, the aircraft has achieved the energy height required to fly at Point B, and so initiates a climb along the constant energy height contour.

The time to climb is determined by numerical integration of equation (6). As a first approximation, the time taken between two points $(\Delta t)$ can be written as

$$
\Delta t=\frac{h_{\mathrm{e} 2}-h_{\mathrm{e} 1}}{\left(P_{\mathrm{s} 1}+P_{\mathrm{s} 2}\right) / 2}
$$

where subscripts 1 and 2 signify the initial and final points, respectively. 


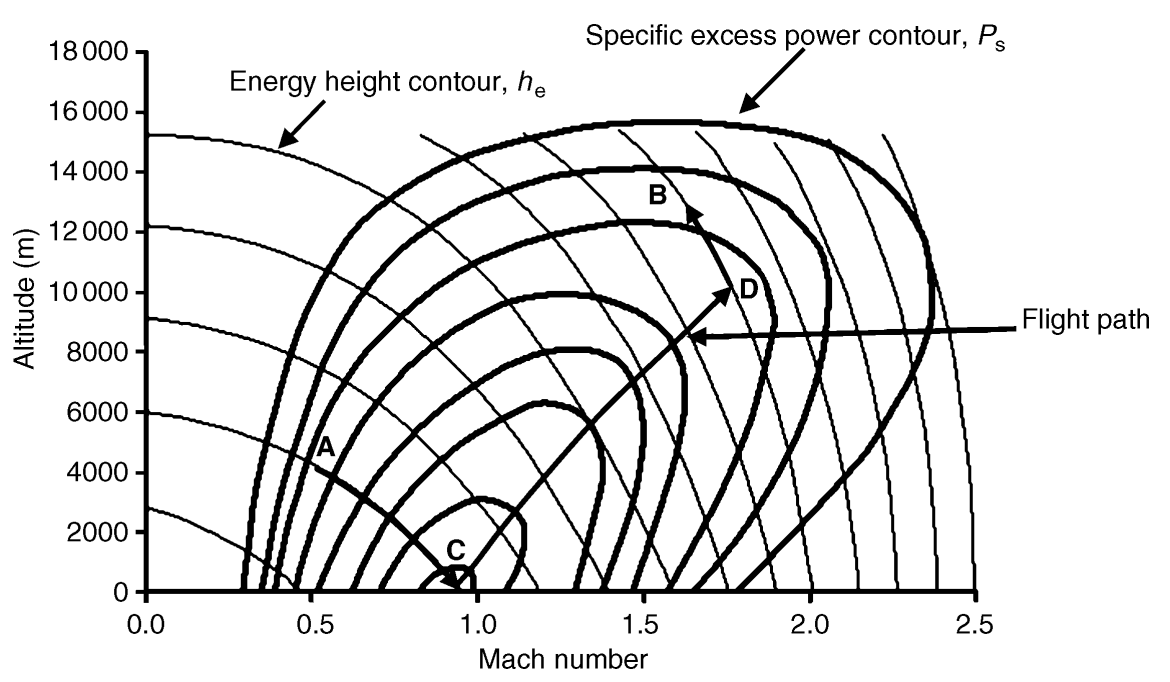

Figure 1. Sketch of an energy climb schedule.

\subsection{Maximum-rate-of-climb schedule vs. maximum-energy-climb schedule}

It is important to recognize that the conditions for maximum climb rate at a given altitude do not necessarily occur on the maximum-energy-climb schedule. Maximizing the climb rate at each altitude gives rise to a maximum-rate-ofclimb schedule. Referring to equations (2) and (3), $P_{\mathrm{s}}$ can be expressed as

$$
P_{\mathrm{s}}=\frac{\mathrm{d} h_{\mathrm{z}}}{\mathrm{d} t}\left(1+\frac{V}{g} \frac{\mathrm{d} V}{\mathrm{~d} h_{\mathrm{z}}}\right)
$$

where $\frac{\mathrm{d} h_{\mathrm{z}}}{\mathrm{d} t}$ is the vertical climb rate $V_{\mathrm{c}}$. To maximise $V_{\mathrm{c}}$ it is necessary to maximize $P_{\mathrm{s}}$ at every altitude rather than the energy height. For a given altitude this will occur when

$$
\frac{\partial P_{\mathrm{s}}}{\partial V}=0
$$

For subsonic aircraft the energy and rate-of-climb schedules are usually similar, although for supersonic aircraft there can be significant differences. A combat aircraft on an intercept mission is not only interested in minimizing the time-to-height, because it will also require a certain speed to carry out the attack. The maximum-energy-climb schedule will minimize the time to achieve the required height and speed. The maximum-rate-of-climb schedule will minimize the time-to-height, but the aircraft will then have to accelerate to the required speed. Particularly at high altitude, the acceleration times can be significant. The maximum-rate-of-climb schedule is used for short climbs, where the time to climb is more important than the final speed.

\section{CLIMB PERFORMANCE}

For combat aircraft, climb performance differs from civil aircraft in two important respects. First, due to high $T / W$ values, climb can occur at high flight path angles. Second, climbs with acceleration are an important combat capability. Therefore, it is necessary to extend the analysis covered earlier (see Volume 5, Chapter eae247).

\subsection{Climbs at high angles}

From Figure 2, for an aircraft in a climb without acceleration

$$
\begin{aligned}
& L=W \cos \gamma \\
& T=D+W \sin \gamma
\end{aligned}
$$

where $\gamma$ is the climb angle. Combining equations (10) and (11) gives

$$
\sin \gamma=\frac{T}{W}-\frac{\cos \gamma}{L / D}
$$

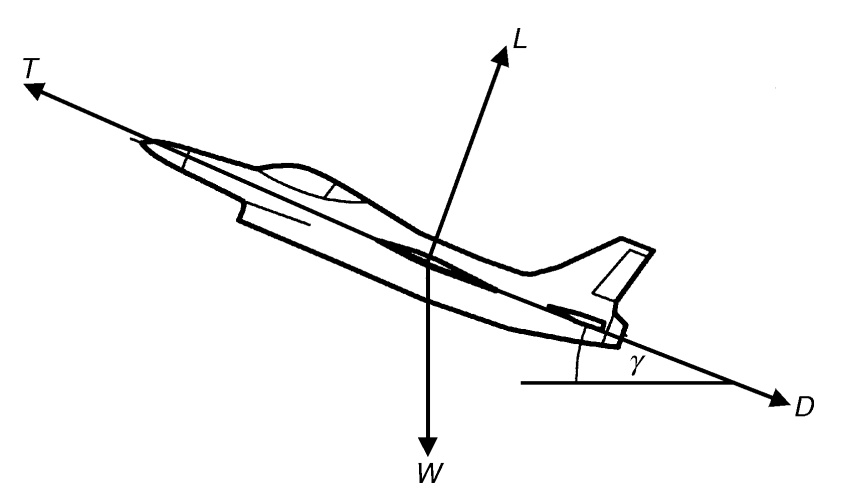

Figure 2. Forces in the climb. 
To maximize $\sin \gamma$, the aircraft should fly at maximum T/W whilst minimizing the effect of the $\frac{\cos \gamma}{L / D}$ term. As $\gamma$ increases, $\cos \gamma$ will reduce, but this effect can be magnified by increasing $L / D$. In other words, to maximize the climb angle, an aircraft should fly at the minimum drag speed and with maximum thrust. This conclusion is consistent with the small climb angle result analysis (see Volume 5, Chapter eae247). However, it is important to recognize that the minimum drag speed changes with climb angle. This is due to the reduction in the induced drag, which is a consequence of the reduced lift required in a climb (equation (10)). The minimum drag speed in a climb is given by modifying the straight and level value (see Volume 5, Chapter eae250)

$$
V_{\mathrm{md}}=\left(\frac{K}{C_{D_{0}}}\right)^{\frac{1}{4}}\left(\frac{W \cos \gamma}{\frac{1}{2} \rho S}\right)^{\frac{1}{2}}
$$

The minimum drag speed reduces as climb angle increases. In the extreme, for a vertical climb the minimum drag speed is zero, since this corresponds to minimum profile drag. Obviously such a result needs to be treated with caution. It should also be noted that equation (12) gives nonsensible results when $T / W$ is greater than 1 . Such cases imply that the aircraft can climb vertically and has the ability to accelerate.

\subsection{Climb with acceleration}

An aircraft's specific excess power can be used for acceleration as well as climbing, or a combination of the two. Referring to equation (2), at any climb condition, the acceleration along the flight path is given by

$$
\frac{\mathrm{d} V}{\mathrm{~d} t}=\frac{g}{V}\left(P_{\mathrm{s}}-\frac{\mathrm{d} h_{\mathrm{z}}}{\mathrm{d} t}\right)
$$

where $\mathrm{d} h_{\mathrm{z}} / \mathrm{d} t$ is the vertical climb velocity $V_{\mathrm{c}}$. For a known combination of air speed and $V_{\mathrm{c}}$, the potential of an aircraft to accelerate can be assessed. At high climb angles it should be noted that from equation (10) the lift, and hence the induced $\mathrm{drag}$, are reduced compared with straight and level flight. The calculation of $P_{\mathrm{s}}$ needs to take this into account.

\section{TURN PERFORMANCE}

Good turn performance in both the horizontal and vertical planes is an important attribute for a combat aircraft. The analysis of the level (horizontal) turn and the vertical pull up carried out for transport aircraft are applicable (see Volume 5 , Chapter eae248), but for combat aircraft there are some important considerations which are not routinely considered by transport aircraft. These considerations include

- The energy loss during instantaneous turns

- Climbing turns

Using the standard turn equations (see Volume 5, Chapter eae248) plots can be constructed to show how the horizontal turn rate $\dot{\psi}$ varies with forward speed $V$. An example plot is shown in Figure 3. For many combat maneuvers the instantaneous turn rate (ITR) is important because it gives the highest turn rate at a given forward speed. The ITR curve has three parts: (i) the thrust limit where the engines are operating at maximum thrust, (ii) the load factor limit which is set by the structural design limits of the aircraft, and (iii) the lift limit which is defined by the onset of stall or buffet. When operating on the ITR an aircraft will always be slowing down, but for an aircraft on the load factor limit the turn rate will increase as forward speed is lost. This increase in turn rate continues until the aircraft reaches the lift limited part of the ITR curve. The speed at which the load factor and lift limited curves meet is called the corner speed, $V_{\text {corner }}$, and gives the condition for maximum ITR. The curve for sustained turn rate (STR) is also shown in Figure 3. Sustained turns are important for combat aircraft, particularly since some modern aircraft are capable of sustaining turns whilst pulling maximum load factors. Such a case is shown in Figure 3. Where the STR intersects the ITR curve, the aircraft can sustain its ITR and will not slow down.

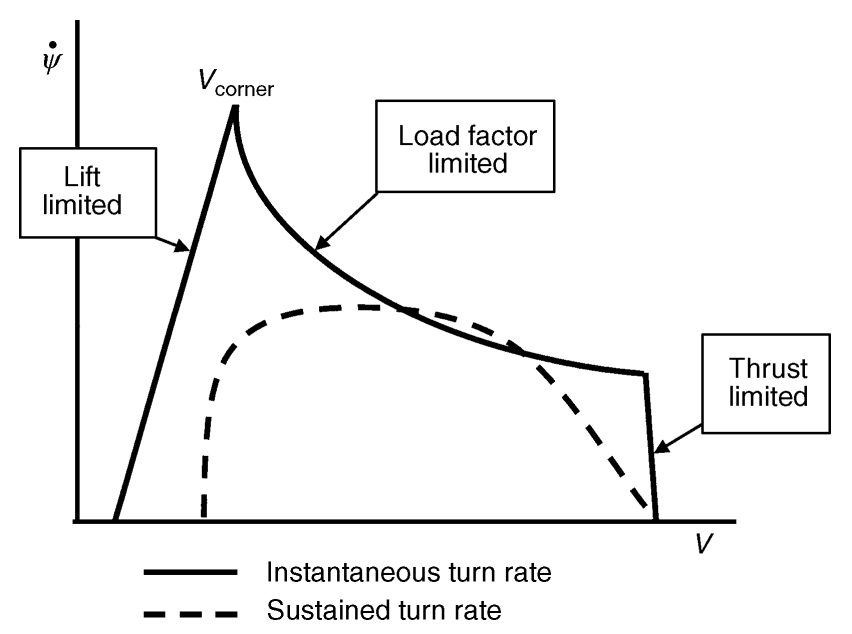

Figure 3. Turn rate plot. 


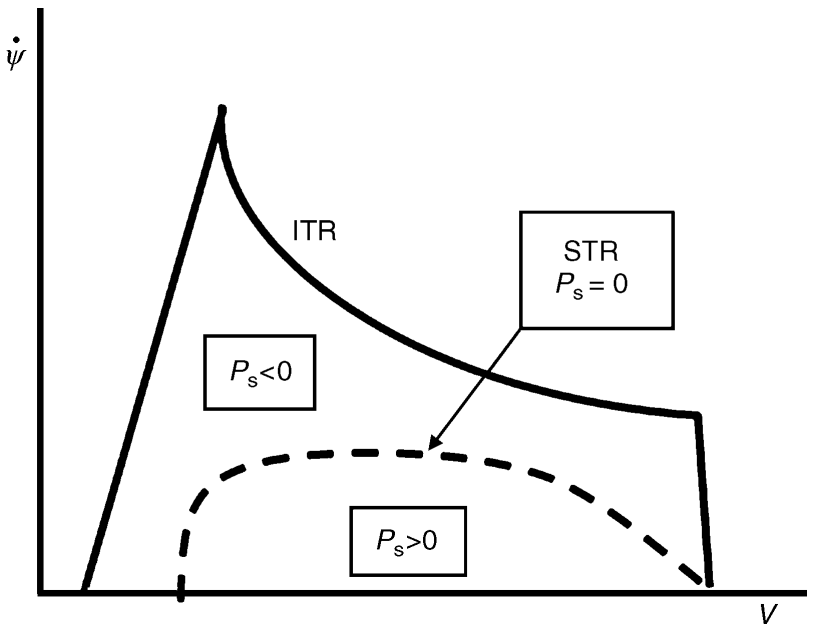

Figure 4. Specific excess power during a horizontal turn.

\subsection{Turn performance in terms of specific excess power}

As already noted, energy and specific excess power $P_{\mathrm{s}}$ are useful concepts for assessing maneuvers of combat aircraft, and they can also be used to analyze turn performance. Consider the horizontal turn rate plot shown in Figure 4, where $\dot{\psi}$ is the angular velocity. On the STR curve $P_{\mathrm{s}}=0$, since thrust equals drag. Within the STR envelope, $P_{\mathrm{s}}$ is greater than zero and. the aircraft can maneuver further without slowing down or losing height (e.g., to a higher turn rate). Above the STR envelope, $P_{\mathrm{S}}$ is less than zero, and the aircraft's speed is always decreasing. This means that an aircraft operating on the ITR curve will always be slowing down and losing energy. Ending a turning maneuver in a low energy state will limit the pilot's options for further maneuvers and can place an aircraft at a disadvantage in a combat engagement.

It is possible to quantify the values of $P_{\mathrm{s}}$. For the load factor limited part of the ITR curve equation (5) applies, but with the thrust set to its maximum value, $T_{\max }$. For the lift limited part of the curve the equation becomes

$$
P_{\mathrm{s}}=V\left(\frac{T_{\max }}{W}-\frac{q S C_{D_{0}}}{W}-\frac{K C_{L_{\max }}^{2} q S}{W}\right)
$$

Knowing $P_{\mathrm{s}}$, the deceleration can be determined from equation (14) with $\mathrm{d} h_{\mathrm{z}} / \mathrm{d} t$ set to zero.

The vertical turn rate plot has a similar form to Figure 3 , and the ITR curve has both load factor and lift limited parts. The forces along the flight path (see Volume 5, Chapter eae248) for the ITR are given by

$$
T_{\max }-D-W \sin \gamma=m \frac{\mathrm{d} V}{\mathrm{~d} t}
$$

which allows the specific excess power to be written as

$$
P_{\mathrm{s}}=\frac{V\left(T_{\max }-D-W \sin \gamma\right)}{W}
$$

Upon expansion of the drag term to include the appropriate form of the induced drag (i.e., $C_{L_{\max }}$ or constant load factor) the resulting equations are identical to equations (5) and (15) except for the addition of $-V \sin \gamma$.

\subsection{The climbing turn}

A climbing turn combines both altitude and heading changes so that the aircraft flies a helical path. The following is based on the approach adopted by Vinh (1993). This is a simplified analysis since the turn is assumed to occur at a constant speed. However, the analysis reveals some interesting insights into the maneuver. Since there is no deceleration, the drag will be relatively low implying a low angle of attack and a moderate load factor. As well as zero acceleration along the flight path, it is assumed that the flight path angle $\gamma$ is constant and that the thrust acts along the $x$-axis. A sketch of the forces acting on the aircraft is shown in Figure 5. These forces are

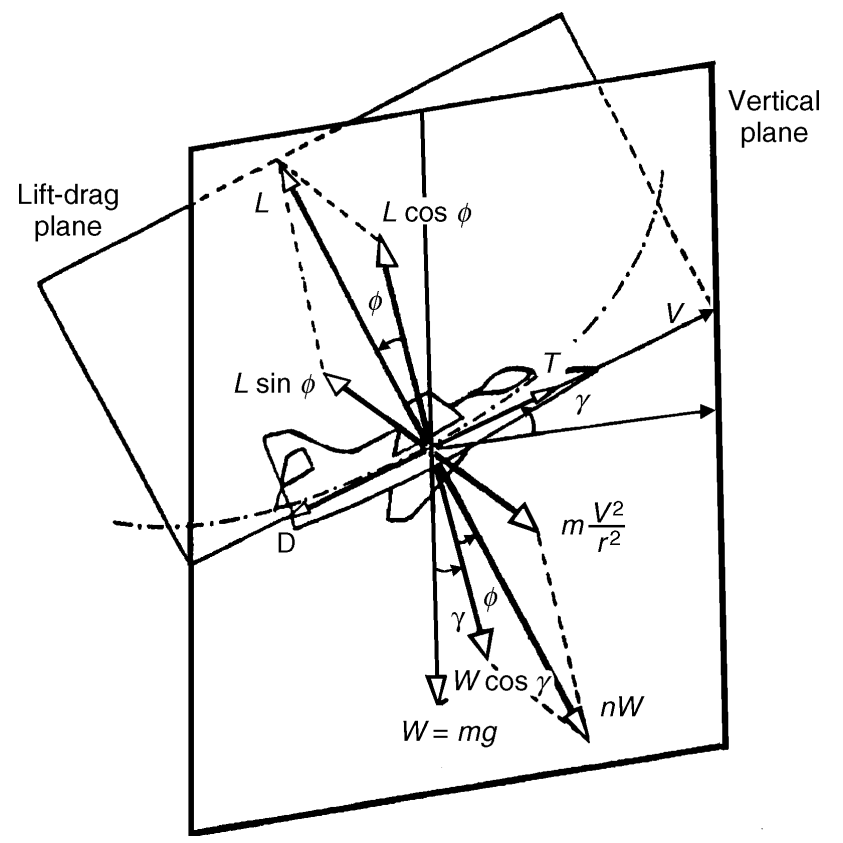

Figure 5. Forces in the climbing turn. Reproduced from Vinh (1993) (c) Cambridge University Press. 
Along the flight path

$$
T=\frac{1}{2} \rho V^{2} S C_{D}+W \sin \gamma
$$

In the vertical plane

$$
L \cos \varphi=W \cos \gamma
$$

Radially

$$
L \sin \varphi=\frac{W}{g} \frac{V^{2}}{r}
$$

where $\varphi$ is the bank angle and $r$ is the radius of the helical turn. When the climb angle $\gamma$ is set to zero, the above equations reduce to those of the horizontal sustained turn (see Volume 5, Chapter eae248). In the climbing turn the definition of load factor, $n=L / W$, is still valid. Inspection of equation (19) shows that the load factor can also be expressed as

$$
n=\frac{\cos \gamma}{\cos \varphi}
$$

For climbing, or descending turns, $\cos \gamma$ will be less than 1 , which implies that the load factor is less than for horizontal turns at the same bank angle.

The rate of change of heading can be obtained from the general kinematic and force equations for a body moving relative to earth axes (see, e.g., Vinh, 1993). The equations can be simplified by assuming a flat earth and ignoring Coriolis acceleration. The resulting equation for the forces in the horizontal plane is

$$
V \frac{\mathrm{d} \psi}{\mathrm{d} t}=n g \frac{\sin \varphi}{\cos \gamma}
$$

where $\mathrm{d} \psi / \mathrm{d} t$ is the rate of change of heading in the horizontal plane. Substituting equation (21) for $n$ gives

$$
\dot{\psi}=\frac{\mathrm{d} \psi}{\mathrm{d} t}=\frac{g}{V} \tan \varphi
$$

which, as expected from horizontal turn analysis, shows that the rate of change of heading is increased by increasing bank angle. equation (21) for load factor can be expanded using trigonometric identities to give

$$
\tan \varphi=\frac{\sqrt{n^{2}-\cos ^{2} \gamma}}{\cos \gamma}
$$

which upon substitution into equation (23) reduces to the turn rate for a horizontal turn when the climb angle, $\gamma$, is zero. It is important to emphasize that equation (23) gives the heading change in a horizontal plane.

The radius of the helical turn can be determined by re-arranging equation (20) to give

$$
r=\frac{V^{2}}{\mathrm{ng} \sin \varphi}
$$

Multiplying equation (24) through by $\cos \varphi$ give

$$
\sin \varphi=\frac{\sqrt{n^{2}-\cos ^{2} \gamma}}{n}
$$

which can then be substituted into equation (25). When the climb angle, $\gamma$, is zero, equation (25) is identical to the radius for a horizontal turn (see Volume 5, Chapter eae248).

The climbing turn can be thought of as a helical path on the surface of a cylinder. For some maneuvers such as a climb away from a ground attack sortie, knowledge of the cylinder's radius $R$ is desirable. From the geometry of a helix, the radius is given by

$$
R=r \cos ^{2} \gamma
$$

For combat maneuvers, a pilot is more concerned with the rate of change of direction of the velocity vector. In moving between two positions along the climbing turn the aircraft will travel a distance s along the arc of a circle. The angle at the center of the arc is the same as the rotation of the velocity vector and is denoted $\chi$. The rate of change of direction of the velocity vector is then

$$
\dot{\chi}=\frac{\mathrm{d} \chi}{\mathrm{d} t}=\frac{1}{r} \frac{\mathrm{d} s}{\mathrm{~d} t}=\frac{V}{r}
$$

Substituting equations (25) and (26) gives

$$
\dot{\chi}=\frac{g}{V} \sqrt{n^{2}-\cos ^{2} \gamma}
$$

Comparing with the combination of equations (23) and (24) leads to the following relationship between changes in velocity vector direction and horizontal heading

$$
\dot{\chi}=\dot{\psi} \cos \gamma
$$

During the climbing turn, it is possible to trade between turn and climb performance. This trade off can be expressed in terms of specific excess power $P_{\mathrm{s}}$. Referring to the forces 
acting along the flight path, equation (18) can be written in terms of $P_{\mathrm{s}}$, by multiplying through by $V / W$

$$
P_{\mathrm{s}}=\frac{T V-\frac{1}{2} \rho V^{3} S C_{D}-W V \sin \gamma}{W}=0
$$

The first term of the numerator, $T V$, is the power available from the engine, the $C_{D}$ term is primarily the power required for the turn and the $\sin \gamma$ term is the power required for the climb. The initial assumptions for the preceding analysis dictate that $P_{\mathrm{s}}$ is zero. For a turn at a given load factor, it is possible to determine the aircraft's speed to maximize the climb rate, $V_{\mathrm{c}}$, where

$$
V_{\mathrm{c}}=V \sin \gamma
$$

Rearranging equation (31) and writing $C_{D}$ in terms of profile and induced drag coefficients gives

$$
V_{\mathrm{c}}=\frac{1}{W}\left[T V-\frac{1}{2} \rho V^{3} S C_{D_{0}}-K \frac{(n W)^{2}}{\frac{1}{2} \rho V S}\right]
$$

To maximize $V_{\mathrm{c}}$ requires $\partial V_{\mathrm{c}} / \partial V=0$. Differentiating equation (33) results in a quadratic equation in $V^{2}$, for which the appropriate root is

$$
V=\sqrt{\frac{T / S}{3 \rho C_{D_{o}}}}\left[1+\frac{12 K C_{D_{0}} n^{2}}{(T / W)^{2}}\right]^{\frac{1}{2}}
$$

Climbing turns are normally only carried out for small altitude changes, so that density $\rho$ and thrust $T$ can be assumed constant. Therefore equation (34) shows that optimum climbing speed increases with load factor

Equation (34) is applicable to a straight (i.e., non-turning) climb. For shallow climb angles, the load factor approximates to 1; but for high angle climbs (see Section 3.1), $n$ is given by equation (21), with $\cos \varphi$ set to 1 . When determining $n$, it is important to note that climb angle $\gamma$ is a function of both $V$ and $V_{\mathrm{c}}$. With the power of modern computers, an iterative numerical analysis can be carried out, until an acceptable degree of accuracy has been attained.

\section{PERFORMANCE METRICS}

Performance metrics are used to allow the combat capabilities of an aircraft to be easily assessed and compared with those of a competitor. Such assessments can be carried out to generate combat techniques, or to help select an aircraft for air force use. An example of a commonly used metric is the turn rate plot, which was shown in Figure 3. This is a powerful metric since, at a given set of conditions, it allows the turn performance of two aircraft to be compared. However, the turn rate plot does have limitations. For example, it does not show how quickly an aircraft will slow down during a turn. It is quite possible that aircraft $\mathrm{A}$ will have a higher turn rate than aircraft $B$ at the start of a turn, but by the end of the turn, B may have the superior performance.

Research into improved and more comprehensive performance metrics is continuing and is being driven by the need to easily quantify and assess the performance advantages of new technologies such as thrust vectoring and post stall maneuvering. Some of these proposed metrics are complicated, and require detailed input data for the subject aircraft. Such data may not be available for enemy aircraft, so there is also a need for accurate metrics based on limited data sets.

The following performance metrics include traditional metrics which are commonly used, and others which, though relatively new, have been adopted by performance engineers. Numerous other metrics have been proposed, and these have been reported in technical literature.

\subsection{Specific excess power plots}

Specific excess power $P_{\mathrm{s}}$ (see Section 2.2) is a powerful tool for comparing aircraft, since at any given condition, the aircraft with the highest $P_{\mathrm{s}}$ has the greater potential to maneuver and is likely to have an advantage in combat. Specific excess power, or SEP as it is frequently called, can be plotted in a number of different ways. For example, plots such as Figure 1 can be produced for two rival aircraft and then compared to establish the altitude-Mach number combinations at which either aircraft has the advantage. An alternative presentation is shown in Figure 6 , where $P_{\mathrm{s}}=0$ contours are plotted for different load factors. To win a combat engagement,

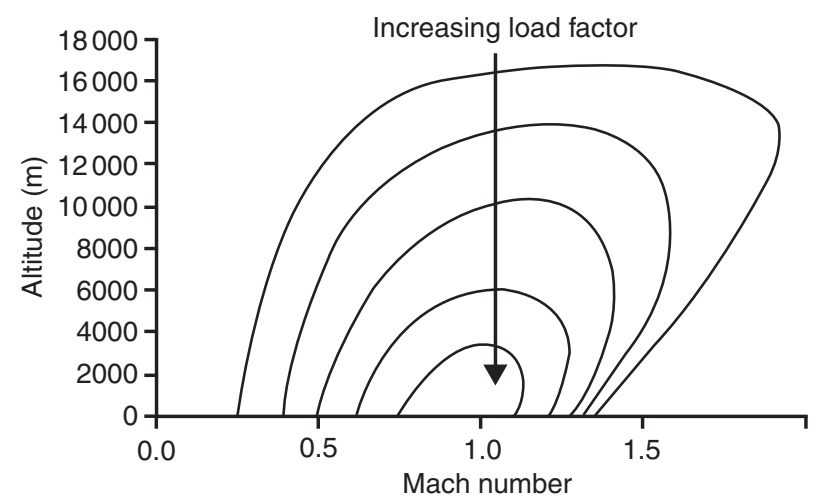

Figure 6. $P_{\mathrm{s}}=0$ Contours for different load factors 
an aircraft's $P_{\mathrm{s}}=0$ contours should envelop those of an opponent, since this implies that the aircraft can match an opponent's maneuvers whilst losing less energy. The problem with SEP plots is that they do not indicate directly the acceleration or turn rates that can be achieved.

\subsection{Dynamic speed turn plot}

The connection between turn rate and specific excess power is discussed in Section 4.1. It is straightforward to superimpose contours of constant $P_{\mathrm{s}}$ onto a horizontal turn rate plot, but a more useful idea is to superimpose contours of constant acceleration (or deceleration). Such contours can be generated using equation (14), with $\frac{\mathrm{d} h_{z}}{\mathrm{~d} t}$ set to zero, to give

$$
\frac{\mathrm{d} V}{\mathrm{~d} t}=\frac{P_{\mathrm{s}} g}{V}
$$

Such a plot is often termed a dynamic speed turn plot, and is useful because it provides additional information that is readily understood by both pilots and designers.

\subsection{Combat cycle time}

A turn rate plot, even when developed into dynamic speed turn plot, only describes what an aircraft is doing at a given point in a turn. It gives no indication of how long an aircraft will take to carry out a specific maneuver (e.g., a turn through $180^{\circ}$ ). It is sometimes suggested that defining a task and carrying it out, is a better way to measure performance than to simply measure, for example, the turn rate and deceleration at a given set of aircraft conditions. Such specific maneuvers are called closed loop tasks.

The concept of a closed loop task can be used to analyse turn performance. As originally defined by Tamrat (1988), the combat cycle time (CCT) is the time to perform a $180^{\circ}$ heading change and return to the original energy state, although the concept is applicable to other maneuvers. A CCT maneuver superimposed on to a turn rate plot is shown in Figure 7. An aircraft flying at $V_{0}$ rolls into a turn, loses speed in the turn and rolls out of the turn at speed $V_{\mathrm{F}}$ when it has turned through $180^{\circ}$. The aircraft then accelerates back to $V_{0}$ to complete the maneuver.

The CCT can be found by summing the times taken for each part of the maneuver. These are

$t_{1}$ time taken for aircraft to bank

$t_{21}$ time taken to slow down to the corner speed $V_{\text {corner }}$

$t_{22}$ time taken from corner speed to complete $180^{\circ}$ heading change

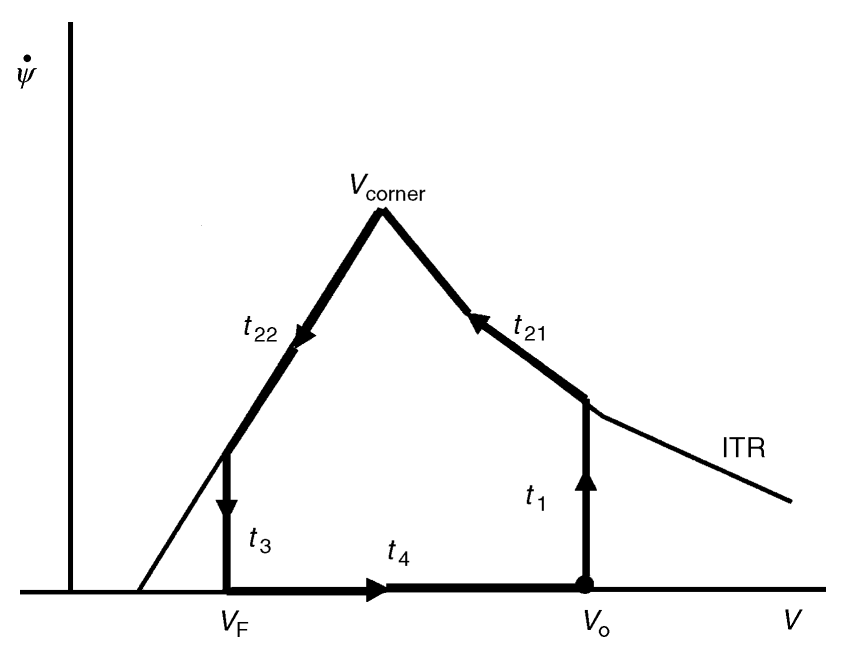

Figure 7. Combat cycle time.

$t_{3}$ time to roll into straight and level flight

$t_{4}$ time for acceleration to original condition

The times taken to roll into and out of the turn $\left(t_{1}, t_{3}\right)$ are often negligible. The times for turning $\left(t_{21}, t_{22}\right)$ are usually determined by numerical integration. The deceleration in the turn is given by equation (35). The time to slow down to corner speed is then given by

$$
t_{21}=\int_{V_{0}}^{V_{\text {comer }}} \frac{V}{P_{\mathrm{s}} g} \mathrm{~d} V
$$

In getting to the corner speed, the aircraft will make a heading change, $\psi_{\mathrm{c}}$, and this can be calculated by noting that he horizontal turn rate is given by

$$
\dot{\psi}=\frac{\mathrm{d} \psi}{\mathrm{d} t}=\frac{g\left(n^{2}-1\right)}{V}
$$

Multiplying equation (37) by elapsed time, gives the heading change

$$
\psi_{\text {corner }}=\sqrt{n^{2}-1} \int_{V_{0}}^{V_{\text {comer }}} \frac{\mathrm{d} V}{P_{\mathrm{S}}}
$$

The calculation for $t_{22}$ is carried out in a similar fashion, but with the added complication that the load factor is changing and is given by

$$
n=\frac{\rho V^{2} S C_{L_{\max }}}{2 W}
$$

The numerical calculation is stopped once the total heading change has reached $180^{\circ}$. If during the turn $P_{\mathrm{s}}$ becomes 
zero, the aircraft has reached a sustained turn and $\dot{\psi}$ is constant. Assuming that the pilot is flying an optimum maneuver, $V_{\mathrm{F}}$ will then become the minimum drag speed.

The final part of the CCT maneuver is an acceleration in level flight, with the time obtained from equation (36) but with the integral limits modified to $V_{\mathrm{F}}$ and $V_{0}$.

\subsection{Pointing margin}

Proposed by Tamrat (1988), the pointing margin is used to assess the ability of an aircraft to point its guns, or other weapon, at an enemy aircraft. Both the enemy and friendly aircraft initiate maximum ITR turns. The maneuver ends when one aircraft can point its weapon at the other aircraft. In Figure 8, this is depicted by the dashed line from the friendly fighter. The pointing margin is defined as the angle between the nose of the adversary and the line of sight of the friendly fighter, at the time when the adversary is aligned with the line of sight. The pointing margin is an angular measurement, but from Figure 8, it can be related to the time taken for the adversary to bring its weapons to bear on the friendly aircraft. Analysis of the pointing margin is essentially similar to that used for the CCT.

Pointing margin can also be used to assess the advantage of adopting post stall maneuvering. technology. For two aircraft making identical opposing turns, the aircraft which can pitch up from the turn to a high angle of attack, will get the first opportunity to point its weapon. The timing of a pitch up

At time $t$, enemy aircraft's

inting in direction

$$
\text { of arrow. }
$$

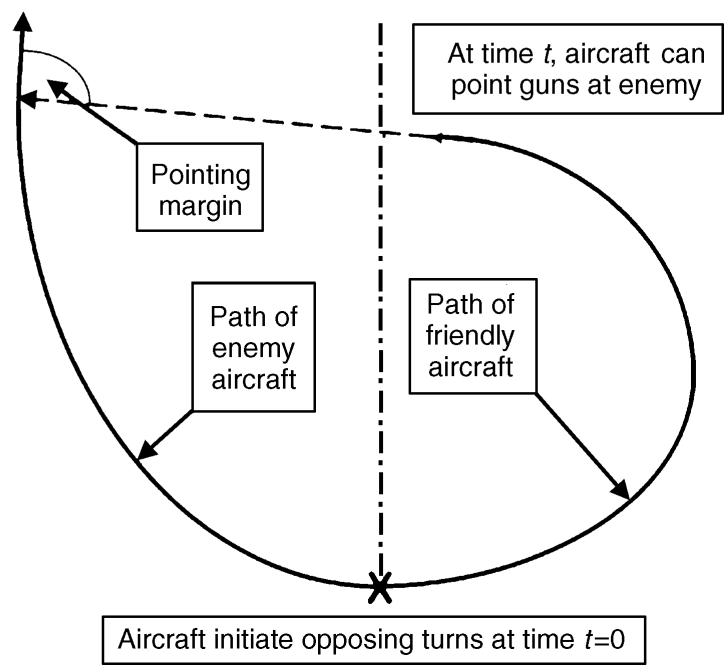

Figure 8. Pointing margin maneuver is critical, since failure to shoot down an opponent will leave an aircraft in a vulnerable low energy state.

\subsection{Kutschera metric}

Whilst attempting to quantify the effects of thrust vectoring and post stall maneuvering, Kutschera and Render (2002) developed a metric which effectively combines pointing margin and CCT.

The metric considers the aircraft flying from an origin to a point where the gun of the aircraft is pointed towards a pre-defined target, meaning that nose pointing is allowed. To reduce the order of complexity the target is assumed to be stationary. Figure 9 shows that for a symmetrical aircraft at the origin, to one side there are seventeen nodes of interest. Each node tests different aspects of the aircraft's performance. For example, node 2 tests the vertical plane performance, whilst node 8 tests the axial acceleration of the aircraft.

Four parameters are used to quantify the aircraft's performance for each node. These are the time taken to complete the maneuver, the SEP of the aircraft at the end of the maneuver, the energy change over the maneuver and the turn diameter Each of these four parameters is important and were identified by combat pilots during interviews conducted by Kutschera. The time taken is required since it is a natural parameter for pilots to discuss. The final SEP is important since it indicates an aircraft's ability to carry out further maneuvers. The energy consumed allows the efficiency of the maneuver to

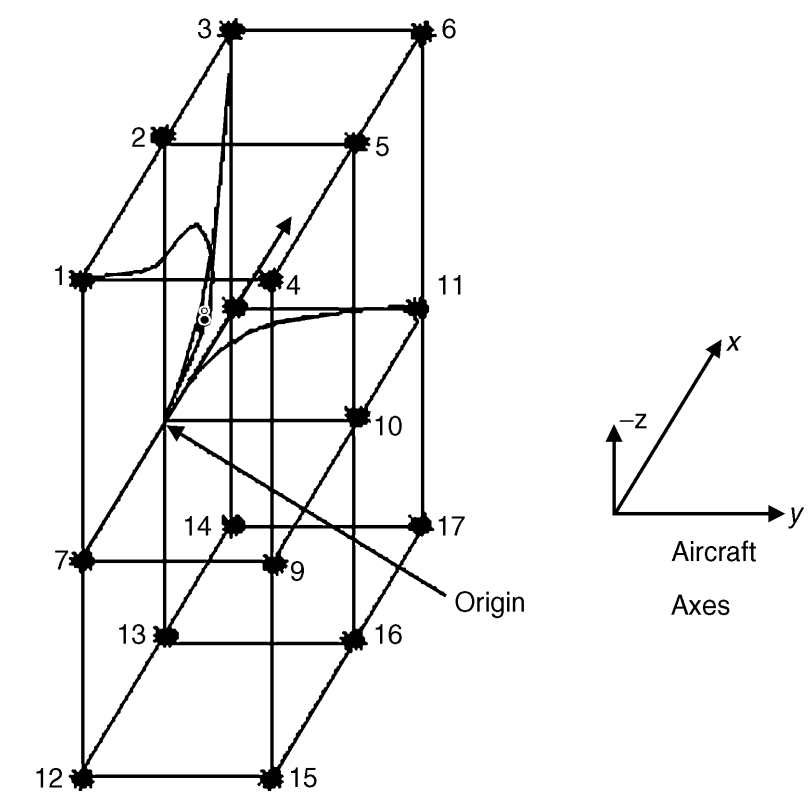

Figure 9. Nodes Used in the Kutschera metric. Reproduced with permission from Kutschera and Render (2002) (C) Royal Aeronautical Society. 

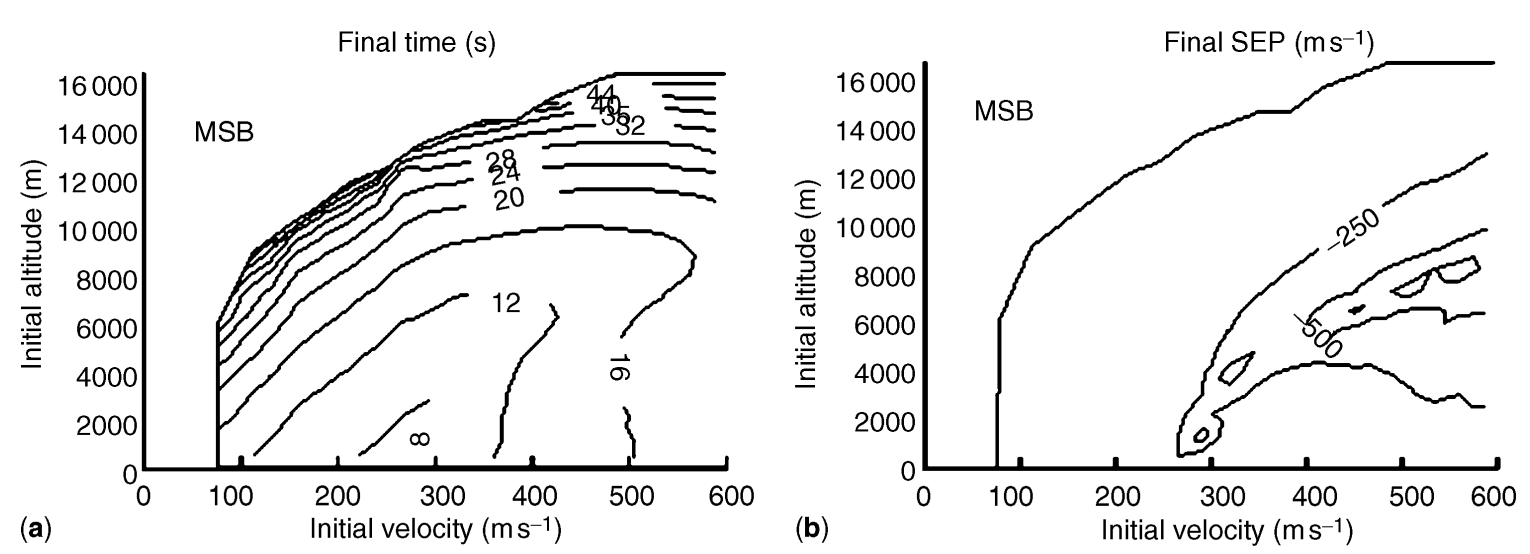

Energy change $(m)$
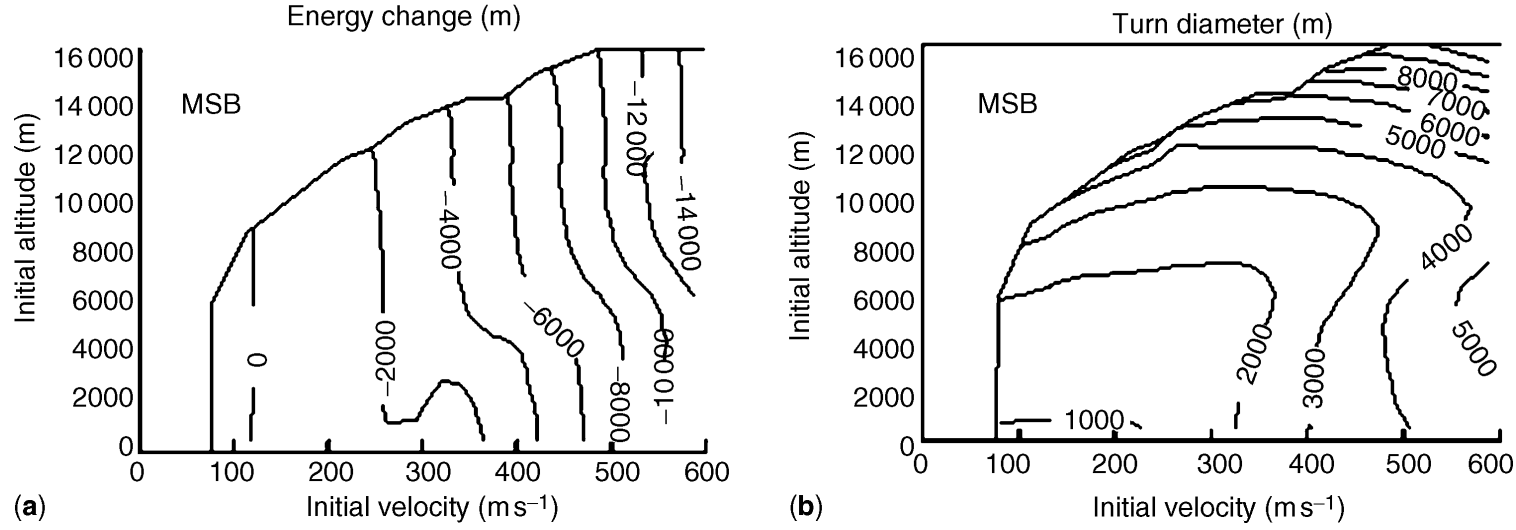

Figure 10. Example outputs from the Kutschera metric for a $180^{\circ}$ heading change. (a) final time taken; (b) final specific excess power; (c) energy change during turn; (d) turn diameter. Reproduced with permission from Kutschera and Render (2002) (c) Royal Aeronautical Society.

be assessed. Finally the turn diameter is given since it gives an idea of the geometry of the maneuver, and allows spatial analysis to be undertaken.

Figure 10 shows results for a Boeing F-18 performing a $180^{\circ}$ horizontal heading change for a number of different altitude and speed combinations. The contours shown have then been generated from the individual results. Heading changes required to face a node (in this case number 7 in Figure 9) can be used to avoid the need to specify a distance from the aircraft to the node. Performance comparisons with other aircraft can be made by plotting the differences instead of the absolute values. MSB refers to the maneuver stall boundary which defines starting conditions where the aircraft stalls during the maneuver.

\section{NEW TECHNOLOGIES}

\subsection{Relaxed stability}

On a conventional aircraft the tailplane is sized to provide trim across the flight envelope.
To maintain trim during both subsonic and supersonic maneuvers the tailplane will produce a download, which acts in opposition to the wing's lift vector. Since the magnitude of an aircraft's overall lift essentially determines its maneuver performance, any loss of lift to overcome tailplane downloads can be viewed as detrimental. The basic longitudinal trim equation (see Volume 5, Chapter eae252) can be written as

$$
0=C_{M_{0}}+C_{L}\left(h-h_{0}\right)-\eta_{T} \bar{V} C_{L_{\mathrm{T}}}
$$

It can be seen that changes in the locations of the center of gravity $h$ and the aerodynamic center $h_{0}$ will influence the value of the tailplane lift coefficient $\left(C_{L_{\mathrm{T}}}\right)$ for trim. In other words, reducing the static stability will reduce the value of $C_{L_{\mathrm{T}}}$. Reducing $C_{L_{\mathrm{T}}}$ gives rise to

- Reduced trim drag and a corresponding improvement in specific excess power which, has been seen, is an important measure of aircraft performance

- The possibility of a smaller tailplane, with a resulting reduction in aircraft weight 
The reduction in stability will also lead to reduced control forces and deflections required to maneuver an aircraft. (see Volume 5, Chapters eae248 and eae252). Modern combat aircraft incorporate flight control systems, which incorporate artificial feel for the pilot. Therefore the reduced control forces and deflections impact upon the design of the control surface actuators.

For the above reasons, relaxed static stability (RSS) is routinely used on modern combat aircraft. The aerodynamic center location of a wing/fuselage combination moves rearwards with increase in Mach number and it is likely that an RSS aircraft will be unstable for part of its flight envelope. A commonly stated myth is that unstable aircraft are uncontrollable by human pilots unless they are assisted by a flight control system. The world's first successful manned aircraft, the Wright Flyer, was deliberately made unstable (Gibbs-Smith, 1985). For manual control, instability means an increased pilot work load since the pilot has to actively oppose the effects of gusts, etc. Increasing the level of instability will result in the more rapid departure of an aircraft from controlled flight, and hence the need for sophisticated flight control systems.

\subsection{Canard configuration}

Some combat aircraft use the canard configuration with a foreplane ahead of the wing replacing the tailplane. As shown in Figure 11, the foreplane produces lift, $L_{\mathrm{F}}$, at a distance $l_{\mathrm{f}}$ in front of the center of gravity. The trim equation becomes

$$
0=C_{M_{0}}+C_{\mathrm{L}}\left(h-h_{0}\right)+\bar{V}_{\mathrm{F}} C_{L_{\mathrm{F}}}
$$

where $C_{L_{\mathrm{F}}}$ is the foreplane lift coefficient and $\bar{V}_{\mathrm{F}}$ is the foreplane volume coefficient defined as

$$
\bar{V}_{\mathrm{F}}=\frac{S_{\mathrm{F}} l_{\mathrm{F}}}{S_{\mathrm{c}}}
$$

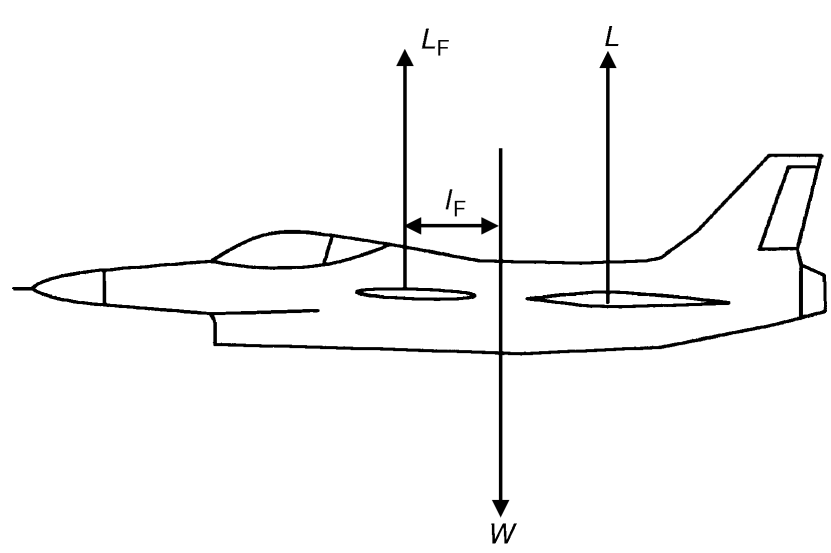

Figure 11. Canard lift arrangement. where $S_{\mathrm{F}}$ is the foreplane area. $S$ and $\overline{\bar{c}}$ are, respectively, wing area and wing aerodynamic mean chord. To achieve inherent stability, the center of gravity of a canard aircraft $h$ lies in front of the aerodynamic center $h_{0}$. With this arrangement the foreplane produces positive lift to trim an aircraft in both subsonic and supersonic maneuvers. The foreplane and wing lift now act in the same direction, giving enhanced maneuvering performance. However, the foreplane will be destabilizing since it generates a nose up pitching moment which, when combined with the forward center of gravity, will result in an unstable aircraft.

On most modern combat aircraft the foreplane and wing are designed to be closely coupled so that there are mutually beneficial interactions between the two surfaces to improve aircraft performance. For example, the vortices and downwash from the foreplane can be used to enhance the lifting characteristics of the wing, particularly to delay the onset of stall and buffet. A commonly cited advantage of the canard arrangement is that it allows good pitch recovery characteristics when the aircraft is at high incidence. This can be achieved by reducing foreplane lift by decreasing incidence, when a conventional tailplane would be required to provide increased lift whilst operating in the separated flow field of the main wing.

\subsection{Thrust vectoring}

Commonly cited advantages of thrust vectoring for combat aircraft include:

- Turn rate enhancement since the thrust can be vectored towards the center of a turn and so increase the turning force.

- Controlled post-stall maneuvers since the vectored thrust provides control forces and moments when conventional aerodynamic surfaces will be stalled.

- Recovery following departure from controlled flight since thrust forces will be available when conventional aerodynamic controls are ineffective.

- Improved take off and landing performance since the thrust is used to supplement the control power.

- Removal or reduction in size of conventional aerodynamic controls. This is usually argued in terms of radar cross section (stealth) or weight reductions.

There are obviously perceived disadvantages with thrust vectoring including, increased weight and complexity, and lack of control redundancy when the engine fails. 
6.3.1 The influence of thrust vectoring on horizontal turns

The following method is based on Raymer (1989) and extends the conventional turn analysis (see Volume 5, Chapter eae248). Consider an aircraft flying a horizontal turn at a bank angle of $\varphi$, with angle of attack $\alpha$ with thrust vectoring deflected through an angle $\varphi_{T}$, the vertical forces now become

$$
\left[L+T \sin \left(\alpha+\varphi_{T}\right)\right] \cos \varphi=W
$$

and the horizontal radial forces become

$$
\left[L+T \sin \left(\alpha+\varphi_{T}\right)\right] \sin \varphi=\frac{m V^{2}}{R}
$$

From conventional turn analysis, the turn rate is given by

$$
\dot{\psi}=\frac{g\left(n^{2}-1\right)^{\frac{1}{2}}}{V}
$$

so to maximize $\dot{\psi}$ for a given speed, it is required to maximize the load factor $n$. The total force acting on the airframe in the lift direction is given by

$$
L+T \sin \left(\alpha+\varphi_{T}\right)=n W
$$

When turning at the structural limit equations (45) and (46) indicate that the addition of thrust vectoring will not increase the turn rate. At speeds below the corner speed, $n$ will be less than the permitted maximum value and thrust vectoring can be used to enhance the turn rate. The load factor increases with $\varphi_{T}$ and the condition to maximize load factor can be determined by differentiating equation (46) with respect to $\varphi_{T}$ and equating to zero. This leads to

$$
\frac{T}{W} \cos \left(\alpha+\varphi_{T}\right)=0
$$

Since $T / W$ is greater than zero, this implies that the thrust vector should be normal to the flight path. As none of the thrust is propelling the aircraft forward it will decelerate rapidly. The BAE Systems/McDonnell Douglas Harrier has used this as a combat technique to make enemy aircraft overshoot, and so provide Harrier pilots with a shooting opportunity.

\section{CONCLUSION}

The preceding sections have described how the maneuver capabilities of fixed-wing combat aircraft can be assessed through the use of straightforward extensions to the analysis techniques used for other types of aircraft. The considered maneuvers have all been essentially steady, and have not considered rapid maneuvering where an aircraft's orientation is abruptly changed. Such maneuvers are likely to excite the dynamic modes of an aircraft and need to be assessed by use of the appropriate equations of motion (see Volume 5, Chapter eae254). The extent to which a combat aircraft can successfully exploit its maneuver performance will depend on many factors including flying and handling qualities (see Volume 5, Chapter eae258).

\begin{tabular}{|c|c|c|}
\hline$\overline{\bar{c}}$ & aerodynamic mean chord & $\mathrm{m}$ \\
\hline СCT & combat cycle time & $\mathrm{s}$ \\
\hline$C_{D_{0}}$ & profile drag coefficient & \\
\hline $\mathrm{C}_{\mathrm{L}}$ & lift coefficient & \\
\hline$C_{L_{\mathrm{F}}}$ & foreplane lift coefficient & \\
\hline$C_{L_{\mathrm{i}}}$ & straight and level lift coefficient & \\
\hline$C_{L_{\max }}^{L_{1}}$ & maximum lift coefficient & \\
\hline$C_{L_{\mathrm{T}}}$ & tailplane lift coefficient & \\
\hline $\begin{array}{l}C_{M_{0}} \\
D\end{array}$ & $\begin{array}{l}\text { pitching moment coefficient at zero lift } \\
\text { drag }\end{array}$ & $\mathrm{N}$ \\
\hline$g$ & acceleration due to gravity & $\mathrm{ms}^{-2}$ \\
\hline$h$ & center of gravity location & \\
\hline$h_{\mathrm{e}}$ & $\begin{array}{l}\text { energy height (specific energy); see } \\
\text { equation (1) }\end{array}$ & $\mathrm{m}$ \\
\hline$h_{\mathrm{z}}$ & height & $\mathrm{m}$ \\
\hline$h_{0}$ & aerodynamic center location & \\
\hline ITR & instantaneous turn rate & $\mathrm{rad} \mathrm{s}^{-}$ \\
\hline$K$ & induced drag constant & \\
\hline$l_{\mathrm{F}}$ & foreplane moment arm & $\mathrm{m}$ \\
\hline$l_{\mathrm{T}}$ & tailplane moment arm & $\mathrm{m}$ \\
\hline$L$ & lift & $\mathrm{N}$ \\
\hline$L_{\mathrm{F}}$ & foreplane lift & $\mathrm{N}$ \\
\hline MSB & maneuver stall boundary & \\
\hline$n$ & load factor $=L / W$ & \\
\hline$P$ & specific power & $\mathrm{ms}^{-1}$ \\
\hline$P_{\mathrm{s}}$ & specific excess power; see equation (3) & $\mathrm{m} \mathrm{s}^{-1}$ \\
\hline$q$ & dynamic pressure & $\mathrm{Nm}^{-2}$ \\
\hline$r$ & helical radius of climbing turn & $\mathrm{m}$ \\
\hline$R$ & horizontal radius of (climbing) turn & $\mathrm{m}$ \\
\hline RSS & relaxed static stability & \\
\hline$s$ & distance along arc of helical flight path & $\mathrm{m}$ \\
\hline$S$ & wing area & $\mathrm{m}^{2}$ \\
\hline$S_{\mathrm{F}}$ & foreplane area & $\mathrm{m}^{2}$ \\
\hline$S_{\mathrm{T}}$ & tailplane area & $\mathrm{m}^{2}$ \\
\hline
\end{tabular}

\section{ACKNOWLEDGMENTS}

Thanks are extended to Goff Tearle and Abdullah Malik for their help in preparing the figures.

\section{NOTATION}


Maneuver of Fixed-Wing Combat Aircraft 13

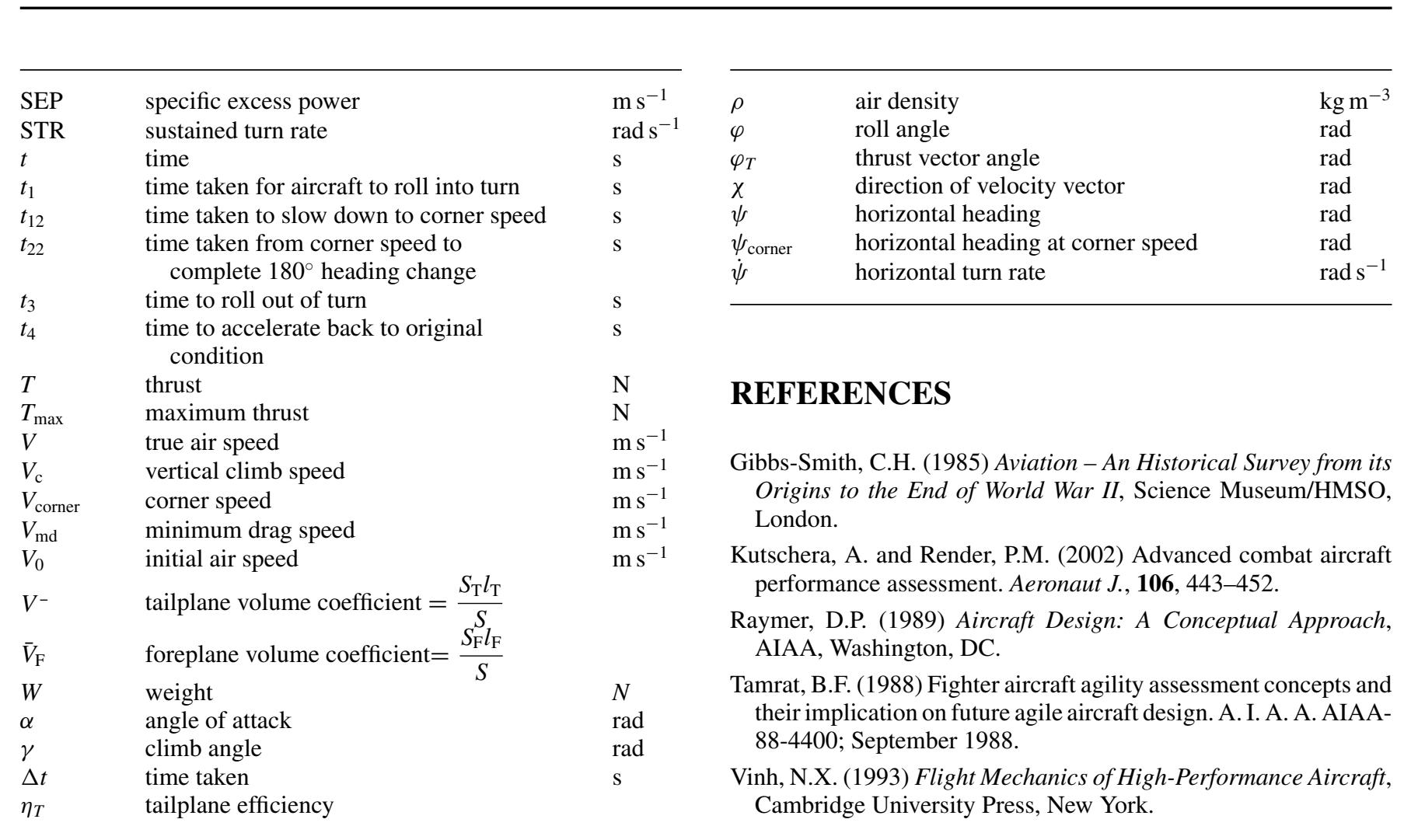




\section{Abstract:}

The importance of energy and specific excess power in assessing the maneuverability of combat aircraft is discussed. This is followed by a discussion on climb and turn performance, which extends the methods presented in other sections. In particular, high angle climbs and the climbing turn are considered. The use of performance metrics to assess aircraft performance is described, and both traditional (e.g., specific excess power plots) and new metrics (e.g., combat cycle time) are described. The article concludes by considering features which have been adopted on the latest combat aircraft, namely relaxed stability, the canard configuration and thrust vectoring.

Keywords: canard, climb, performance metrics, relaxed stability, specific excess power, thrust vectoring, turning 
Author Query

Q1 Please varify the unit of measurement of final SEP and Energy change is parts (b) and (c) of Figure 10 respectively. We think units for final specific excess power (SEP) and energy change should be something else other than provide the in artwork. 